\title{
Development and Bio-imaging Applications of a Chemical Imaging Sensor
}

\author{
Ko-ichiro Miyamoto ${ }^{1 *}$ and Tatsuo Yoshinobu ${ }^{1,2}$ \\ ${ }^{1}$ Department of Electronic Engineering, Tohoku University, \\ 6-6-5 Aza-Aoba, Aramaki, Aoba-ku, Sendai, Miyagi 980-8579, Japan \\ ${ }^{2}$ Department of Biomedical Engineering, Tohoku University, \\ 6-6-5 Aza-Aoba, Aramaki, Aoba-ku, Sendai, Miyagi 980-8579, Japan
}

(Received February 24, 2016; accepted June 14, 2016)

Keywords: chemical imaging sensor, label-free, light-addressable potentiometric sensor

A chemical imaging sensor is a field-effect-based semiconductor sensor, capable of label-free imaging of ion distributions in a solution. The sensor has attracted much interest owing to its simple structure and diverse fields of application of bioimaging. In this article, the principle and development of the chemical imaging sensor are summarized, compared with those of other labelfree imaging sensors. Bio-imaging applications of the chemical imaging sensor to ion diffusion, enzymatic reaction, and metabolic activities of microorganisms are also reviewed.

\section{Introduction}

Microscopic imaging with an optical microscope is the most fundamental observation technique in biology. Among all such techniques, fluorescent microscopy, which is able to selectively visualize the molecules of interest, has been the most essential tool. Labeling with a fluorescent dye, however, may disturb the functionality of target biomolecules, and the toxicity of the dye and color degradation may be problems in some applications.

Label-free imaging techniques based on electrochemical measurement using electrodes and/or semiconductor sensors have been studied to overcome this problem. Although the spatial resolution is still poor compared with optical methods in general, these techniques have attracted much interest owing to their potential for label-free imaging.

Label-free sensor devices for chemical imaging can be classified into the two categories of "arrayed" and "nonarrayed" imaging sensors, as shown in Table 1. An arrayed sensor of the first category is realized as an array of individual electrodes or sensors, each corresponding to a predefined pixel. With the help of semiconductor microfabrication technology, a large number of tiny electrodes or sensors can be integrated onto a single chip. The measurement system can be miniaturized by on-chip integration of peripheral circuits such as amplifiers. Some of these devices are already available on the market.

A nonarrayed sensor of the second category has a monolithic structure of the semiconductor sensor plate and relies on the light addressing technique for the definition of pixels and spatially resolved measurement.

"Corresponding author: e-mail: k-miya@ecei.tohoku.ac.jp 
Table 1

Arrayed and nonarrayed image sensors.

\begin{tabular}{lcc}
\hline & Arrayed sensor & Nonarrayed sensor \\
\hline $\begin{array}{l}\text { Principle } \\
\text { Spatial resolution }\end{array}$ & MEA/IS-FET/CCD-based & LAPS \\
Layout of pixels & $\begin{array}{c}\text { Pimited by pixel layout } \\
\text { Medefined by circuit design }\end{array}$ & Limited by carrier diffusion \\
Measurement area & $($ e.g., $1024 \times 1024)$ & Flexible \\
Process/Cost & Chip size & (e.g., $256 \times 256)$ \\
& (order of $\left.\mathrm{mm}^{2}\right)$ & Wafer size \\
Complicated $/$ Expensive & $\left.\mathrm{cm}^{2}\right)$ \\
\end{tabular}

In this article, both arrayed and nonarrayed imaging sensors are described. In Sect. 2, the measurement principles and bio-imaging applications of arrayed sensors are briefly reviewed. In Sect. 3, we focus on the chemical imaging sensor, which is a nonarrayed imaging sensor. The principle and development to improve the sensitivity and spatial resolution of the chemical imaging sensor are described. In Sect. 4, several bio-imaging applications of the chemical imaging sensor are summarized.

\section{Arrayed Imaging Sensor}

An arrayed imaging sensor consists of an array of electrodes or semiconductor sensors for chemical/bio-imaging. Figure 1 shows diagrams of four arrayed imaging sensors. The first example of this kind is a microelectrode array (MEA). The density of electrodes has increased rapidly with the help of microfabrication technology, and the functionality has been enhanced by the integration of additional circuits. A large-scale integration-based amperometric sensor (BioLSI) is an array of electrodes, each of which functions individually as an amperometric sensor. An ion-sensitive fieldeffect transistor (IS-FET) array is an LSI with an array of semiconductor-based chemical sensors and peripheral circuits for rapid readout of the current signal. A CCD/CMOS-based ion imaging sensor is an LSI with an array of potential wells to store a charge and peripheral circuits to read out the charge as a signal responding to the Nernst potential. Table 2 lists the properties of the MEA, BioLSI, IS-FET array, and CCD/CMOS-based ion imaging sensor.

The feasibility of devices with a huge number of pixels and the possibility of fast readout for a high frame rate are the common advantages of arrayed imaging sensors. Devices with $512 \times 512$ or $1024 \times 1024$ pixels have already been realized with CMOS technology. On the other hand, a device of this kind tends to be expensive because of the high cost of microfabrication. Good passivation of the circuit elements and wires is still required to protect them from water and chemical substances in the analyte and enable stable and long-term use, as the standard process does not consider the use of the semiconductor device in contact with chemicals.

\subsection{Microelectrode array}

A MEA was originally designed to record electrical activities of a neuronal network. ${ }^{(1-3)}$ As depicted in Fig. 1(a), it started with a simple structure of a planar metal pattern, and developed into a more sophisticated device with integrated circuits for measurement. The density of electrodes has been drastically increased. For instance, arrays of $64 \times 64^{(4)}$ and $128 \times 128^{(5)}$ electrodes with 
pitches of 21 and $7.8 \mu \mathrm{m}$, respectively, are reported. As the electrodes are miniaturized, reduction of the input impedance becomes more important to obtain a good $\mathrm{S} / \mathrm{N}$ ratio of the signal. Different materials have been tested, such as gold, platinum, and iridium alloy. Also, porous materials, fibers and carbon nanotubes are used to increase the net surface area. ${ }^{(6)}$ Not only the density of electrodes but also the frame rate has been developed; Johnson et al. reported $20 \times 10^{3} \mathrm{fps}$ with an array of $28 \times 40$ (7) $^{(7)}$ As a recent example of its application, the propagation of the action potential along the axon of a single neuron was observed using a MEA device. ${ }^{(8)}$

\subsection{BioLSI}

The BioLSI is an array of amperometric sensors proposed by Inoue et al. ${ }^{(9)}$ The device consists of $20 \times 20$ microelectrodes with a $250 \mu \mathrm{m}$ spacing, and as shown in Fig. 1(b), each is functionalized with an in-pixel amplifier for measurement at a temporal resolution of $18 \mathrm{~ms} / \mathrm{image}$. By coating the sensor surface with gel polymer containing horseradish peroxidase, the production and diffusion of $\mathrm{H}_{2} \mathrm{O}_{2}$ catalyzed by glucose oxidase was clearly visualized.(9) Reported applications of the BioLSI includes the characterization of an embryoid body by monitoring the activity of alkaline phosphatase, ${ }^{(10)}$ the visualization of dopamine release from spheroid cells, ${ }^{(11)}$ and conductivity imaging. ${ }^{(12)}$

\subsection{IS-FET array}

The IS-FET is a semiconductor-based chemical sensor invented by Bergveld in $1970{ }^{(13)}$ with some essential modifications made by Matsuo and Wise. ${ }^{(14)}$ Figure 1(c) is a schematic view of its structure, which is similar to that of a conventional MOSFET, except that the IS-FET has no gate metal and the insulator surface is in contact with a solution. The variation of the Nernst potential on the insulator surface of an IS-FET, which is a function of the activity of the target ion or the $\mathrm{pH}$ of the solution, is detected in the form of a drain current. ${ }^{(15)}$ A compact $\mathrm{pH}$ meter with the IS-FET is widely available in the market. The advantages of the IS-FET include (1) high compatibility with the standard fabrication processes for semiconductors, (2) ease of miniaturization, and (3) stability and ease of use compared with a glass $\mathrm{pH}$ electrode. Thanks to these advantages, IS-FETs have been used in many applications. ${ }^{(16-19)}$

With the help of the microfabrication technologies, IS-FET arrays have been developed for chemical/bio-imaging applications. Martinoia et al. fabricated an array of 12 IS-FETs for monitoring cellular metabolism and an integrated device with an area of $5 \times 5 \mathrm{~mm}^{2}$ consisting of 40 IS-FETs and 20 microelectrodes for the electrophysiological measurement of neurons. ${ }^{(20)}$ Milgrew et al. fabricated a $16 \times 16$ array of IS-FETs by CMOS technology. ${ }^{(21,22)}$

In recent years, highly integrated IS-FET arrays fabricated with CMOS technology have been reported. Nemeth et al. reported a movie recording of $\mathrm{pH}$ change using an array of $64 \times 64$ ISFETs fabricated with the $0.35 \mu \mathrm{m}$ process rule. ${ }^{(23)}$ A $0.18 \mu \mathrm{m}$ process rule was used in several studies between 2010 and 2014. Chan et al., ${ }^{(24)}$ Shields et al..(25) and Huang et al. ${ }^{(26)}$ fabricated arrays of $16 \times 16$ and $64 \times 64$ IS-FETs. The IS-FET array reported in Ref. 26 was designed with a pixel pitch of $10 \mu \mathrm{m}$ and could yield a recorded movie of the $\mathrm{pH}$ change at $1200 \mathrm{fps}$. In 2015, Jiang et al. fabricated an array of $512 \times 575$ IS-FETs with a pixel pitch of $4.4 \mu \mathrm{m}$ and achieved movie recording at $375 \mathrm{fps}^{(27)}$ The feasibility of an array with a huge number of pixels at a high density and the possibility of fast data readout with a high frame rate are the main advantages of an IS-FET array. 


\section{$2.4 \mathrm{CCD} / \mathrm{CMOS}-\mathrm{based} \mathrm{pH}$ imaging sensor}

Sawada et al. developed another type of device that measures the charge responding to the Nernst potential, using the charge transfer technology of a CCD. ${ }^{(28)}$ Figure 1(d) shows a unit cell of the sensor corresponding to one pixel. The pixel has a sensing area and a potential well, the depth of which responds to the Nernst potential. An input diode and an input control gate are used to fill the potential well with charge, and then a floating diffusion and a transfer gate are used to transfer the charge to be measured. The number of pixels and their density have been increased: $32 \times 32$ pixels with a pitch of $130 \mu \mathrm{m},{ }^{(29)} 128 \times 128$ pixels with a pitch of $23 \mu \mathrm{m},{ }^{(30)}$ and $1024 \times$ 1024 pixels with a pitch of $12.1 \mu \mathrm{m}^{(31)}$ have been reported. In addition to $\mathrm{pH}$, the concentration of other ions such as $\mathrm{K}^{+(29)}$ and $\mathrm{Na}^{+(32)}$ and biomolecules such as acetylcholine ${ }^{(33,34)}$ have been measured by modifying of the sensor area. A multimodal sensor, which simultaneously measures an optical image and a $\mathrm{pH}$ image, was also proposed. ${ }^{(35,36)}$ The feasibility of a megapixel device and the possibility of multimodal imaging are the advantages of a $\mathrm{CCD} / \mathrm{CMOS}$-based $\mathrm{pH}$ image sensor.

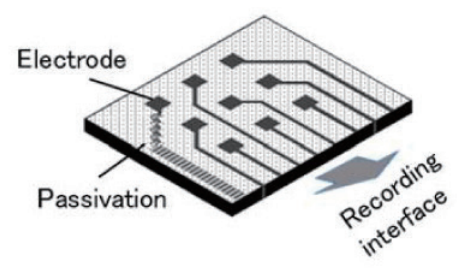

(a)

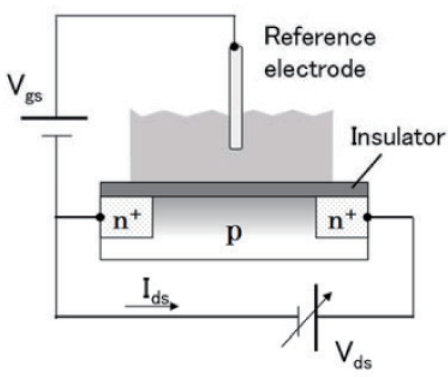

(c)

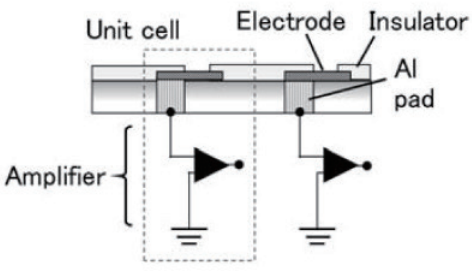

(b)

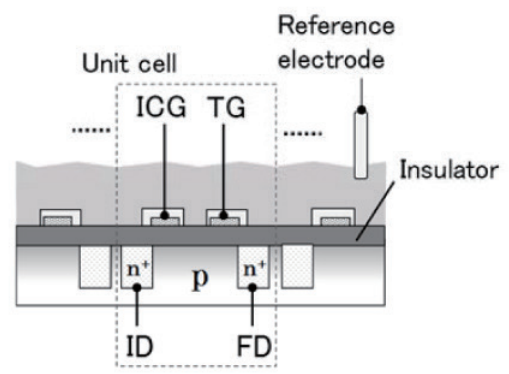

(d)

Fig. 1. Basic structures of (a) microelectrode array, ${ }^{(1-3)}$ (b) BioLSI, ${ }^{(9)}$ (c) ion-sensitive field-effect transistor, ${ }^{(13-15)}$ and (d) $\mathrm{CCD} / \mathrm{CMOS}$-based $\mathrm{pH}$ imaging sensor. ${ }^{(33)}$

Table 2

Overview of arrayed image sensors.

\begin{tabular}{lcccc}
\hline $\begin{array}{l}\text { Highest performance } \\
\text { reported }\end{array}$ & $\begin{array}{c}\text { Micro-electrode } \\
\text { array }\end{array}$ & BioLSI & $\begin{array}{c}\text { IS-FET } \\
\text { array }\end{array}$ & $\begin{array}{c}\text { CCD-based } \\
\text { pH image sensor }\end{array}$ \\
\hline Pixel & $128 \times 128$ & $20 \times 20$ & $512 \times 575$ & $1024 \times 1024$ \\
Pitch of pixels & $7.8 \mu \mathrm{m}$ & $250 \mu \mathrm{m}$ & $4.4 \mu \mathrm{m}$ & $12.1 \mu \mathrm{m}$ \\
Frame rate (fps) & $20 \mathrm{k} \mathrm{fps}$ & $55 \mathrm{fps}$ & $1200 \mathrm{fps}$ & $58 \mathrm{fps}$ \\
& $@ 28 \times 40$ pixels $^{(7)}$ & $@ 20 \times 20$ pixels $^{(9)}$ & $@ 64 \times 64$ pixels $^{(25)}$ & $@ 128 \times 128$ pixels $^{(29)}$ \\
\hline
\end{tabular}




\section{Nonarrayed Imaging Sensor}

In this section, we focus on the chemical imaging sensor based on the principle of the lightaddressable potentiometric sensor (LAPS) proposed by Hafeman et al. ${ }^{(37)}$ In contrast to arrayed imaging sensors, the chemical imaging sensor has no predefined pixels, and a pixel is defined at the time of measurement by illuminating the sensor plate. The sensor plate itself has no built-in device structures or wires, which makes this sensor advantageous in terms of fabrication cost and flexibility of pixel layout. On the other hand, it requires optics and a scanning mechanism, which hinders miniaturization and limits the frame rate of imaging.

In the following subsections, the measurement principle and the construction of chemical imaging sensor systems are briefly explained. After that, the development of its performance and applications are overviewed.

\subsection{Measurement principle of LAPS}

A LAPS has the stacking structure of electrolyte-insulator-semiconductor (EIS), analogous to that of an IS-FET. A DC bias is applied to the EIS system, so that a depletion layer is formed in the semiconductor layer. The width and the capacitance of the depletion layer are dependent on the DC bias and the Nernst potential at the sensing surface of the insulator, which is a function of the activity of target ions. To detect the variation of the depletion layer, the sensor plate is illuminated with a blinking light, which excites photocarriers in the semiconductor layer. When the light is turned on, electrons and holes are generated at the back of the sensor plate and they diffuse toward the front. Electrons and holes are separated by the field inside the depletion layer, resulting in a transient current charging its capacitance. When the light is turned off, a transient current flows in the reverse direction. Figure 2(a) shows the equivalent circuit of the sensor. This is a parallel circuit of capacitances of the depletion layer $\left(C_{\mathrm{d}}\right)$ and that of the insulator $\left(C_{\mathrm{i}}\right)$. The AC source $\left(I_{\text {photo }}\right)$ represents cyclic diffusion of photocarriers and charge separation. $I_{\mathrm{ac}}$ is recorded as a sensor signal.

A current-voltage $(I-V)$ curve is obtained by measuring the amplitude of the alternating photocurrent in the external circuit as a function of the DC bias applied to the sensor. The variation

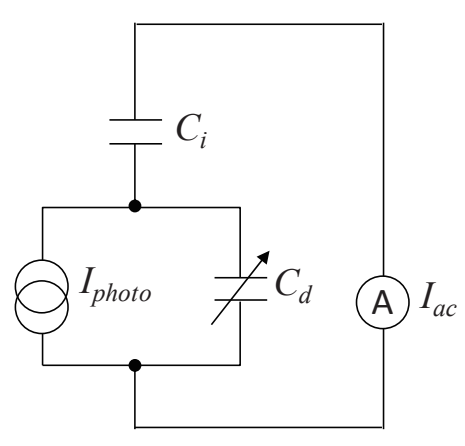

(a)

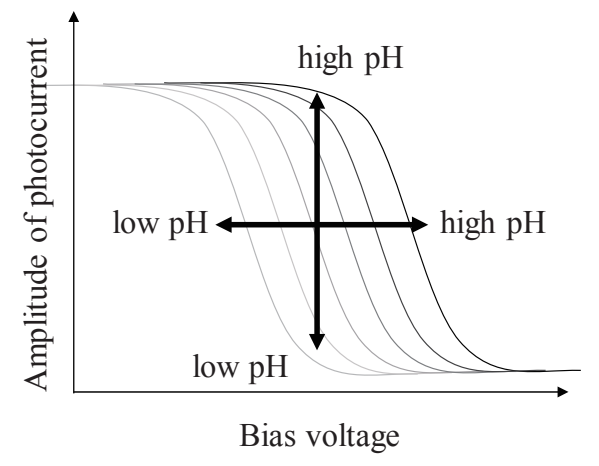

(b)

Fig. 2. (a) Equivalent circuit of LAPS. (b) Schematic view of the $I-V$ curve and its response to the changes in $\mathrm{pH}$ value. 
of the Nernst potential, and therefore, of the activity of target ions, can be determined by measuring the shift of the $I-V$ curve, as shown in Fig. 2(b). Here, the measured area on the sensor surface is defined by illumination, or more precisely, by the extent to which photocarriers reach via diffusion in the semiconductor layer. A detailed analysis based on a device simulator was carried out by Guo et al. ${ }^{(38)}$ and provided a clear view of carrier diffusion.

\subsection{Measurement principle of chemical imaging sensor}

The significance of light addressability was emphasized in the original paper. ${ }^{(37)}$ By fully taking advantage of the light addressability of LAPS, Nakao et al. proposed a chemical imaging sensor in 1994. ${ }^{(39,40)}$

A chemical imaging sensor can visualize the two-dimensional distribution of ions by acquiring a photocurrent map using a scanning laser beam. In most cases, a chemical imaging sensor is operated in the constant-bias mode, in which the amplitude of alternating photocurrent is measured at each pixel under a fixed DC bias condition, because it is too time-consuming to acquire all the $I-$ $V$ curves to determine the potential shift at all pixels. A linear relationship between the DC bias and the amplitude of alternating photocurrent is assumed in the vicinity of the inflection point of the $I-$ $V$ curve, at which the DC bias is set, and the variation of current is converted into a potential shift, which is further converted into the variation of the ion concentration or $\mathrm{pH}$ value. In this way, a chemical image is acquired in a practical length of time.

In contrast to an arrayed imaging sensor, the chemical imaging sensor has no limitations, in principle, on the number of pixels. The extremely simple structure of the sensor plate is advantageous for its low cost, especially in applications where disposable sensors are preferred. The completely flat sensor surface may be advantageous, for example, in applications to microfluidic devices.

\subsection{Measurement system of chemical imaging sensor}

The measurement system of the chemical imaging sensor consists of a sensor plate, a light source, a measurement well and an external circuit, as depicted in Fig. 3.

The sensor plate is made of Si. Both n-type and p-type Si can be used. Typical values of the thickness and resistivity of the Si plate are 200-500 $\mu \mathrm{m}$ and $10 \Omega \cdot \mathrm{cm}$, respectively. A thermal oxide layer about $50 \mathrm{~nm}$ thick is formed on $\mathrm{Si}$, followed by the second insulating layer about $100 \mathrm{~nm}$ thick. The second insulating layer functions as an ion-sensitive layer and also as a passivation layer against the penetration of ions into the oxide layer, which may cause a drift of the sensor signal. ${ }^{(41)}$ After removing the insulating layers on the back of the sensor plate, gold or aluminum electrodes are evaporated near the edges of the sensor plate to form ohmic contacts.

The sensor surface is brought into contact with the analyte solution accommodated in a measurement well. In a standard electrochemical setup, a reference electrode (RE) and a counter electrode (CE) are immersed in the solution, and the potential of the sensor plate as a working electrode (WE) is controlled with respect to the RE, by a potentiostat, and the AC of the WE is measured as the sensor signal. Since no DC flows through the EIS system, a simplified setup is possible, in which the $\mathrm{CE}$ is connected to a bypass capacitor and a DC bias with respect to the RE is applied to the WE without using a potentiostat. 


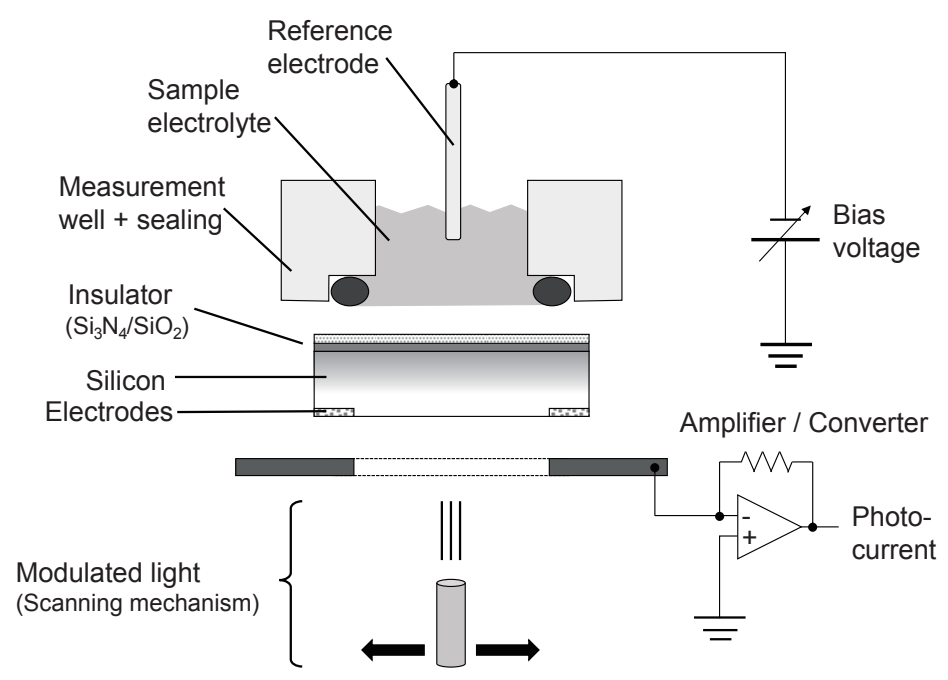

Fig. 3. Measurement system of LAPS and chemical imaging sensor.

A light source blinking at a frequency of approximately $3 \mathrm{kHz}$ is placed underneath the sensor plate to excite photocarriers in the semiconductor. In a typical setup of a chemical imaging sensor, focusing optics and a scanning mechanism are employed to scan the sensor plate with a focused light beam. A transimpedance amplifier is inserted between the ohmic contact of the sensor plate and the ground to measure the $\mathrm{AC}$ of the WE. An $I-V$ curve is obtained by measuring the amplitude of $\mathrm{AC}$ as a function of the DC bias applied to the WE. A chemical image in the constant-bias mode is obtained by recording the amplitude of $\mathrm{AC}$ as a function of the illuminated position under a fixed DC bias condition.

\subsection{Development of chemical imaging sensor}

There have been various efforts to enhance the performance of chemical imaging sensor systems in various aspects.

Correction of artifact signal: There are several different origins of artifact signals that appear in a chemical image. For example, a striation defect in the Si wafer results in a concentric stripe pattern in the obtained chemical image, owing to a reduction in the photocurrent as a result of the enhanced recombination of carriers at defects. ${ }^{(42,43)}$ The distribution of the flat-band potential due to contamination of the sensor surface is another origin of artifact. To overcome these problems, a calibration procedure was proposed.(44) Two types of calibration maps, which correct for distributions of photocurrent and flat-band potential, are prepared for an individual sensor plate prior to measurement.

The phase-mode operation ${ }^{(45)}$ was proposed as a robust mode of measurement. In this mode, the phase of the alternating photocurrent signal with respect to the modulation of illumination is acquired. Compared with the conventional amplitude mode, in which the amplitude of the alternating photocurrent signal is acquired, the phase mode is much less sensitive to the loss of photocarriers at defects as well as to the fluctuation of the light source. 
Another type of artifact originates from the nonlinearity of the $I-V$ curve. In the constantbias mode, the amplitude of the alternating photocurrent signal under a fixed DC bias condition is measured and converted into the potential shift, assuming a linear relationship between the DC bias and the alternating photocurrent. In reality, the alternating photocurrent saturates at its maximum or minimum for a large variation of the ion concentration or $\mathrm{pH}$. In such cases, the shift of the $I-V$ curve should be determined at all pixels. ${ }^{(46)}$

High-speed imaging: The total time required to obtain a chemical image is dependent on the sampling time per pixel, the interval between two pixels for a mechanical scan, and the number of pixels. For instance, the acquisition time of a chemical image with $128 \times 128$ pixels is typically in the range of 3-10 min assuming $10 \mathrm{~ms}$ of sampling time per pixel.

In 2001, Zhang et al. proposed multipoint measurement, in which different locations on the sensor plate are simultaneously illuminated by multiple light beams modulated at different frequencies. ${ }^{(47)}$ The AC photocurrent signal obtained as a superposition of respective frequencies can be separated by Fourier analysis. Compared with a sequential scan using a single light beam, the total time can be reduced several-fold.

Miyamoto et al. used a linear array of 16 LEDs to scan the sensor plate one-dimensionally and acquired a chemical image with $16 \times 128$ pixels in 6.4 s. ${ }^{(48)}$ An array of vertical-cavity surface emitting lasers (VCEL) was also employed to define the pixels at a higher density. ${ }^{(49)}$ The multiplicity was raised to 64, and an optical fiber bundle was used to deliver light beams from 64 LEDs to corresponding pixels densely arranged on the sensor plate. ${ }^{(50,51)}$ A movie recording at a resolution of $8 \times 8$ pixels and a frame rate of $200 \mathrm{fps}$ was realized. The use of an optical fiber bundle has another advantage that the pixel layout can be flexibly changed.

For the generation of modulation signals, programmable oscillators, ${ }^{(47,48)}$ and a phase locked loop (PLL) circuit ${ }^{(50)}$ have been used. A field-programmable gate array (FPGA) is advantageous not only for the generation of modulation signals in many channels but also for real-time Fourier analysis of the signal. ${ }^{(49,52)}$

High spatial resolution: The spatial resolution of a chemical imaging sensor is determined by the size of the area in which the capacitance of the depletion layer is charged with photocarriers. This area is determined by the size of the illuminated spot and the length of the lateral diffusion of photocarriers inside the semiconductor layer. A device simulation ${ }^{(38,53)}$ gives a clear view of the influence of various parameters on the spatial resolution. Thinning of the Si substrate ${ }^{(40,54)}$ and the use of an infrared light beam that enters deeper into $\mathrm{Si}^{(55)}$ are two simple methods of enhancing the spatial resolution. A thinned sensor plate, however, is fragile and difficult to handle.

Another approach is to use a semiconductor material with a shorter diffusion length, such as $\mathrm{GaAs}^{(56)}$ or amorphous $\mathrm{Si} .{ }^{(57,58)}$ Moritz et al. employed amorphous $\mathrm{Si}$ and demonstrated a high spatial resolution in the submicron range. ${ }^{(58)}$ Compared with the sensors based on single-crystalline $\mathrm{Si}$, however, the stability of an insulating layer on these semiconductor materials is still a problem. Krause et al. employed silicon-on-insulator (SOI) and silicon-on-sapphire (SOS) with diffusion lengths of 13 and $0.6 \mu \mathrm{m}$, respectively. ${ }^{(59)} \mathrm{A}$ self-assembled monolayer was used instead of an oxide layer and a spatial resolution higher than $2 \mu \mathrm{m}$ was demonstrated. ${ }^{(60,61)}$

Other methods have been proposed to spatially confine the generation and diffusion of photocarriers to obtain a high spatial resolution. Krause et al. employed a femtosecond laser with a wavelength of $1560 \mathrm{~nm}$ focused into the space charge region. ${ }^{(59)}$ Photocarriers are generated only 
in the proximity of the focal point by the two-photon effect. It was demonstrated that the spatial resolution was improved by $31 \%$ compared with the case of an ordinary HeNe laser. Miyamoto et al. proposed a donut-shaped constant illumination surrounding a blinking light beam. ${ }^{(62)}$ The constant illumination increases the carrier concentration in the surrounding zone, and blocks the lateral diffusion of photocarriers by enhancing recombination. A device simulation predicts an improvement in the spatial resolution by about $40 \%{ }^{(63)}$

Miniaturization of the measurement system: A conventional measurement system for a chemical imaging sensor is typically large in size because of the focusing optics and scanning mechanism. To realize a portable system, a drastic reduction of the system is indispensable.

Miyamoto et al. ${ }^{(64)}$ developed a miniaturized system based on an organic light-emitting diode (OLED) display panel, the measurement speed of which was enhanced by Werner et al. ${ }^{(65)}$ An OLED panel was placed directly underneath the sensor plate and a blinking pixel displayed on the panel scanned the sensor plate to obtain a chemical image with up to $288 \times 64$ pixels.

Wagner et al. ${ }^{(66)}$ developed a miniaturized system based on a digital micromirror device (DMD). A DMD is an array of a huge number of micromirrors fabricated with MEMS technology. By switching the angles of individual micromirrors reflecting the light, a huge number of pixels on the sensor plate can be individually addressed. Das et al. ${ }^{(67)}$ employed 2-axis scanning micromirrors for rapid scanning of the sensor plate with a laser beam. A chemical image with $500 \times 400$ pixels was scanned in $40 \mathrm{~s}$. Taking advantage of the large number of pixels, a zoom-in function was demonstrated both in a DMD-based system ${ }^{(68)}$ and in a scanning-micromirror-based system. ${ }^{(69)} \mathrm{A}$ large area is initially scanned with a sparse set of pixels, and then an area of interest is gradually zoomed-in on with an increasing density of pixels.

\section{Application of Chemical Imaging Sensor}

This section is an overview of the applications of the chemical imaging sensor. The first example is the observation of ion diffusion, which is a fundamental functionality required for various chemical and bio-imaging applications. The second example is an application of $\mathrm{pH}$ imaging to the visualization of enzymatic reactions. The third example is the visualization of the acidification as a result of the metabolic activity of microorganisms.

\subsection{Ion diffusion}

Yoshinobu et al. visualized ion diffusion between an anode and a cathode in the course of the electrolysis of $0.01 \mathrm{M} \mathrm{NaCl}$ solution. ${ }^{(70)}$ The spreading of acidic and anodic regions around electrodes was fitted with a diffusion equation to determine the diffusion coefficients, and a dependence of the diffusion coefficient on the molecular weight was observed.

A chemical imaging sensor was also applied to the visualization of $\mathrm{pH}$ distribution inside a microfluidic channel. ${ }^{(71-73)}$ A laminar flow in a Y-shaped channel and ion diffusion across the boundary were visualized to determine the diffusion coefficient. ${ }^{(73)}$ Figure 4 shows photocurrent distributions inside the Y-shaped channel, in which ion diffusion between different flow rates of laminar flows were observed. The chemical imaging sensor is expected to be the platform for novel microfluidic devices that detect the mass-dependent diffusion across the laminar flow boundary. ${ }^{(74,75)}$ 


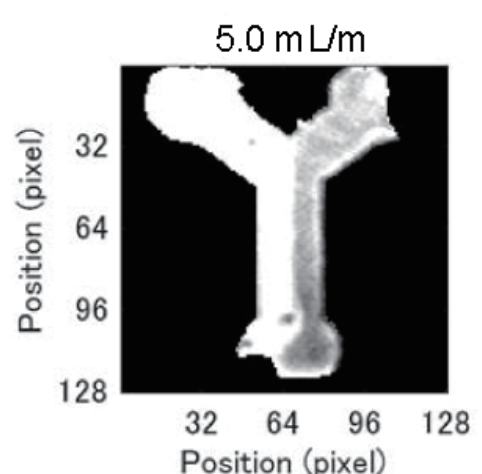

(a)

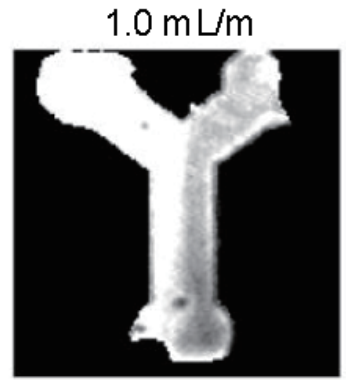

(b)

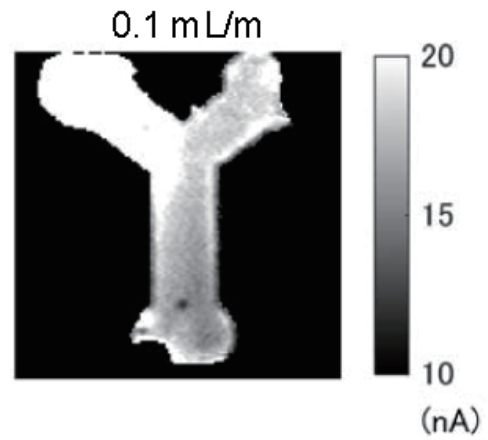

(c)

Fig. 4. Visualization of diffusion between laminar flows with different flow rates of (a) 5.0, (b) 1.0, and (c) $0.1 \mathrm{~mL} / \mathrm{h}$. (Modified from Ref. 73)

\subsection{Enzymatic reaction}

The chemical imaging sensor has also been applied to biosensing, in which the product of an enzymatic reaction was detected. ${ }^{(72)}$ Miyamoto et al. reported the visualization of enzymatic reaction in a microfluidic channel, in which microbeads of gel containing urease were trapped inside the channel and the $\mathrm{pH}$ change in response to the injection of urea was detected downstream. ${ }^{(76)}$ Figures 5(a) and 5(b) show the trap structure and typical chemical images inside the channel, respectively. The potential distribution depends on the flow rate, which corresponds to the reaction time of enzymatic reaction.

The chemical imaging sensor was also applied to the visualization of the neurotransmitter, acetylcholine. Werner et al.(77) applied a chemical imaging sensor to the visualization of acetylcholine by modifying the sensor surface with acetylcholine esterase. The visualization of acetylcholine with a CCD-based $\mathrm{pH}$ imaging sensor has also been reported. ${ }^{(33,34,77)}$

\subsection{Microorganism}

As examples of applications to cells and microorganisms such as bacteria, the metabolic activity of yeast colonies ${ }^{(39)}$ and Escherichia coli (E. Coli) colonies $^{(55,78)}$ have been studied. By culturing microorganisms on an agar plate to form bacterial colonies and measuring the $\mathrm{pH}$ distribution inside the agar plate using the chemical imaging sensor, the $\mathrm{pH}$ change in the surroundings of the colonies caused by metabolic products can be detected. Compared with the conventional plate-counting method, a chemical imaging sensor can not only count the number of colonies but also quantify the metabolic activities of individual colonies.

On the other hand, the measurement of the cellular potential or charges on cellular membranes is not well studied. The electrostatic screening of the charges of ions, biomolecules, and cells in aqueous solution, especially under physiological conditions, is the main problem. While the distance between the charged sample and the sensor surface should be less than $1 \mathrm{~nm}$, ${ }^{(79)}$ it is known that a typical distance between a cell membrane and the substrate is in the range of 10 to $100 \mathrm{~nm} .{ }^{(80)}$ 


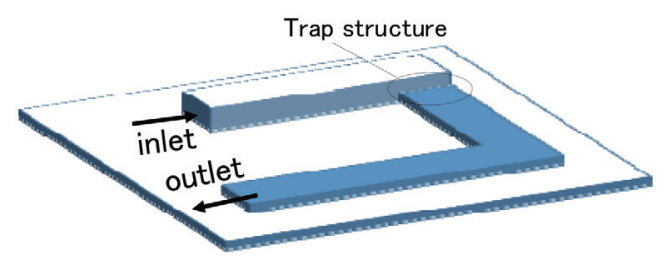

(a)
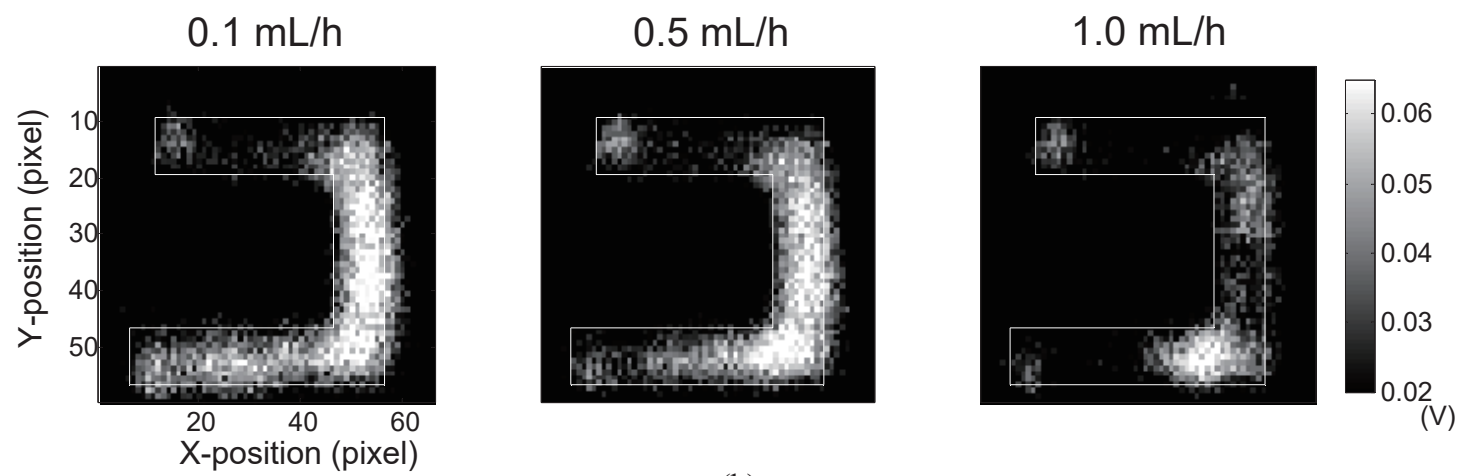

(b)

Fig. 5. (Color online) (a) Schematic view of the channel fabricated on the chemical imaging sensor with a trap structure of the micro-gel beads. (b) Changes in potential distribution depending on the injection rate of the sample. (Modified from Ref. 76)

In the measurement of charged DNA molecules using an LAPS or EIS capacitive sensor, ${ }^{(81-83)} \mathrm{a}$ low-ionic-strength environment was used to mitigate the shielding effect. For further applications of a chemical imaging sensor to cell measurement, it is necessary to overcome the problem of the shielding effect.

\section{Conclusions}

In this review, arrayed and nonarrayed chemical/bio-imaging sensors were overviewed. An arrayed sensor consists of small elements such as electrodes and transistors. The number and the density of pixels of arrayed imaging sensors have been enhanced with the help of microfabrication technology, and various applications have been reported, showing their great potential for labelfree bioimaging. It is important to understand the requirement of the application in order to choose an adequate type of sensor. If a high frame rate or a large number of pixels is necessary, the arrayed imaging sensor would fit the application. On the other hand, a nonarrayed imaging sensor would be suitable for an application that requires flatness of the sensor surface for cell culture and flexible definitions of the measurement area and pixels.

The development and applications of the chemical imaging sensor were also reviewed. The chemical imaging sensor is a nonarrayed imaging sensor based on the light-addressing technique, and has the advantage of a flexible definition of pixels at the time of use. It has been applied to the visualization of ion diffusion, enzymatic reaction, and metabolic activities of microorganisms.

Further development of the spatial resolution, measurement speed, and accuracy of both types of imaging sensors is desired to advance their applications in label-free bio-imaging. 


\section{References}

1 C. A. Thomas Jr, P. A. Springer, G. E. Loeb, Y. Berwald-Netter, and L. M. Okun: Exp. Cell Res. 74 (1972) 61.

2 G. W. Gross, E. Rieske, G. W. Kreutzberg, and A. Meyer: Neurosci. Lett. 6 (1977) 101.

3 M. E. J. Obien, K. Deligkaris, T. Bullmann, D. J. Bakkum, and U. Frey: Front. Neurosci. 8 (2015).

4 L. Berdondini, K. Imfeld, A. MacCione, M. Tedesco, S. Neukom, M. Koudelka-Hep, and S. Martinoia: Lab Chip 9 (2009) 2644.

5 B. Eversmann, M. Jenkner, F. Hofmann, C. Paulus, R. Brederlow, B. Holzapfl, P. Fromherz, M. Merz, M. Brenner, M. Schreiter, R. Gabl, K. Plehnert, M. Steinhauser, G. Eckstein, D. Schmitt-Landsiedel, and R. Thewes: IEEE J. Solid-State Circuits 38 (2003) 2306.

6 X. J. Huang, A. M. OMahony, and R. G. Compton: Small 5 (2009) 776.

7 B. Johnson, S. T. Peace, T. A. Cleland, and A. Molnar: Biomedical Circuits and Systems Conference (BioCAS), 2013 IEEE (2013) p. 109

8 D. J. Bakkum, U. Frey, M. Radivojevic, T. L. Russell, J. Müller, M. Fiscella, H. Takahashi, and A. Hierlemann: Nat. Commun. 4 (2013) 2181.

9 K. Y. Inoue, M. Matsudaira, R. Kubo, M. Nakano, S. Yoshida, S. Matsuzaki, A. Suda, R. Kunikata, T. Kimura, R. Tsurumi, T. Shioya, K. Ino, H. Shiku, S. Satoh, M. Esashi, and T. Matsue: Lab Chip 12 (2012) 3481.

10 M. Şen, K. Ino, K. Y. Inoue, T. Arai, T. Nishijo, A. Suda, R. Kunikata, H. Shiku, and T. Matsue: Biosens. Bioelec. 48 (2013) 12.

11 H. Abe, K. Ino, C. Z. Li, Y. Kanno, K. Y. Inoue, A. Suda, R. Kunikata, M. Matsudaira, Y. Takahashi, H. Shiku, and T. Matsue: Anal. Chem. 87 (2015) 6364.

12 Y. Kanno, K. Ino, K. Y. Inoue, M. Şen, A. Suda, R. Kunikata, M. Matsudaira, H. Abe, C. Z. Li, H. Shiku, and T. Matsue: J. Electroanal. Chem. 741 (2015) 109.

13 P. Bergveld: IEEE Trans. Biomed. Eng. BME-17 (1970) 70.

14 T. Matsuo and K. D. Wise: IEEE Trans. Biomed. Eng. BME-21 (1974) 485.

15 J. Janata: Principles of Chemical Sensors, 2nd ed. (Springer, New York, 2009).

16 B. H. van der Schoot and P. Bergveld: Biosensors 3 (1987) 161.

17 P. Bergveld: Sens. Actuators, B 88 (2003) 1.

18 S. Joo and R. B. Brown: Chem. Rev. 108 (2008) 638.

19 C. S. Lee, S. Kim, and M. Kim: Sensors 9 (2009) 7111.

20 S. Martinoia, N. Rosso, M. Grattarola, L. Lorenzelli, B. Margesin, and M. Zen: Biosens. Bioelec. 16 (2001) 1043.

21 M. J. Milgrew, M. O. Riehle, and D. R. S. Cumming: Sens. Actuators, B 111-112 (2005) 347.

22 M. J. Milgrew, M. O. Riehle, and D. R. S. Cumming: Solid-State Circuits Conference, 2008 (ISSCC 2008) Digest of Technical Papers, IEEE International (2008) p. 590.

23 B. Nemeth, M. S. Piechocinski, and D. R. S. Cumming: Sens. Actuators, B 171-172 (2012) 747.

24 W. P. Chan, B. Premanode, and C. Toumazou: IEEE J. Solid-State Circuits 45 (2010) 1923.

25 P. Shields, B. Nemeth, R. B. Green, M. O. Riehle, and D. R. S. Cumming: IEEE Sens. J. 12 (2012) 2744.

26 X. Huang, F. Wang, J. Guo, M. Yan, Y. Hao, and K. S. Yeo: 2014 Symposium on VLSI Circuits, Digest of Technical Papers (2014) p. 1.

27 Y. Jiang, X. Liu, X. Huang, J. Guo, M. Yan, H. Yu, J. C. Huang, C. H. Hsieh, and T. T. Chen: IEEE Custom Integrated Circuits Conference 2015 (CICC) (2015) p. 1.

28 K. Sawada, S. Mimura, K. Tomita, T. Nakanishi, H. Tanabe, M. Ishida, and T. Ando: IEEE Trans. Electron Devices. 46 (1999) 1846.

29 T. Hattori, Y. Masaki, K. Atsumi, R. Kato, and K. Sawada: Anal. Sci. 26 (2010) 1039.

30 M. Futagawa, D. Suzuki, R. Otake, F. Dasai, M. Ishida, and K. Sawada: IEEE Trans. Electron Devices. 60 (2013) 2634.

31 M. Futagawa, R. Otake, F. Dasai, M. Ishida, and K. Sawada: 18th International Conference on Solid-State Sensors, Actuators and Microsystems (Transducers 2015) (2015) p. 236.

32 T. Hattori, M. Yoshitomo, S. Mori, D. Miyamoto, R. Kato, and K. Sawada: Electroanal. 24 (2012) 114.

33 S.-R. Lee, M. M. Rahman, K. Sawada, M. Ishida, and K. Sawada: Trends Anal. Chem. 28 (2009) 196.

34 S. Takenaga, Y. Tamai, K. Okumura, M. Ishida, and K. Sawada: Jpn. J. Appl. Phys. 51 (2012) 027001.

35 K. Sawada, T. Ohshina, T. Hizawa, H. Takao, and M. Ishida: Sens. Actuators, B 106 (2005) 614.

36 H. Nakazawa, M. Ishida, and K. Sawada: IEEE Sens. J. 11 (2011) 2718.

37 D. G. Hafeman, J. W. Parce, and H. M. McConnell: Science 240 (1988) 1182. 
49 T. Wagner, C. F. B. Werner, K. Miyamoto, M. J. Schöning, and T. Yoshinobu: Sens. Actuators, B 154 (2011) 124.

50 A. Itabashi, N. Kosaka, K. I. Miyamoto, T. Wagner, M. J. Schöning, and T. Yoshinobu: Sens. Actuators, B 182 (2013) 315.

51 K. Miyamoto, A. Itabashi, T. Wagner, M. J. Schöning, and T. Yoshinobu: Sens. Actuators, B 194 (2014) 521.

52 T. Wagner, C. F. Werner, K. Miyamoto, H. J. Ackermann, T. Yoshinobu, and M. J. Schöning: Phys. Status Solidi A 207 (2010) 844.

53 Y. Guo, K. Miyamoto, T. Wagner, M. J. Schöning, and T. Yoshinobu: Sens. Actuators, B 204 (2014) 659.

54 H. Yu, J. Wang, Q. J. Liu, W. Zhang, H. Cai, and P. Wang: Biosens. Bioelec. 26 (2011) 2822.

55 M. Nakao, S. Inoue, T. Yoshinobu, and H. Iwasaki: Sens. Actuators, B 34 (1996) 234.

56 W. Moritz, I. Gerhardt, D. Roden, M. Xu, and S. Krause: Fresenius J. Anal. Chem. 367 (2000) 329.

57 J. C. van den Heuvel, R. C. van Oort, and M. J. Geerts: Solid State Commun. 69 (1989) 807.

58 W. Moritz, T. Yoshinobu, F. Finger, S. Krause, M. Martin-Fernandez, and M. J. Schöning: Sens. Actuators, B 103 (2004) 436.

59 S. Krause, W. Moritz, H. Talabani, M. Xu, A. Sabot, and G. Ensell: Electrochim. Acta 51 (2006) 1423.

60 J. Wang, F. Wu, M. Watkinson, J. Zhu, and S. Krause: Langmuir 31 (2015) 9646.

61 J. Wang, Y. Zhou, M. Watkinson, J. Gautrot, and S. Krause: Sens. Actuators, B 209 (2015) 230.

62 K. Miyamoto, K. Seki, Y. Guo, T. Wagner, M. J. Schöning, and T. Yoshinobu: Proc. Eng. 87 (2014) 612.

63 Y. Guo, K. Seki, K. Miyamoto, T. Wagner, M. J. Schöning, and T. Yoshinobu: Appl. Phys. Express. 7 (2014).

64 K. Miyamoto, K. Kaneko, A. Matsuo, T. Wagner, S. Kanoh, M. J. Schöning, and T. Yoshinobu: Sens. Actuators, B 170 (2012) 82.

65 C. F. Werner, T. Wagner, K. I. Miyamoto, T. Yoshinobu, and M. J. Schöning: Sens. Actuators, B 175 (2012) 118.

66 T. Wagner, K. Miyamoto, C. F. Werner, M. J. Schöning, and T. Yoshinobu: Sens. Lett. 9 (2011) 812.

67 A. Das, T.-C. Chen, C.-M. Yang, and C.-S. Lai: Sens. Actuators, B 198 (2014) 225.

68 T. Wagner, K. Miyamoto, C. F. Werner, M. J. Schöning, and T. Yoshinobu: 16th Int. Solid-State Sens. Actuators, Microsyst. Conf. (Transducers 2011) (2011) p. 2133.

69 A. Das, C. M. Yang, T. C. Chen, and C. S. Lai: Vacuum 118 (2015) 161.

70 T. Yoshinobu, T. Harada, and H. Iwasaki: Jpn. J. Appl. Phys. Part 2-Letters 39 (2000) L318.

71 T. Yoshinobu, H. Iwasaki, Y. Ui, K. Furuichi, Y. Ermolenko, Y. Mourzina, T. Wagner, N. Näther, and M. J. Schöning: Methods 37 (2005) 94.

72 K. Miyamoto, M. Yoshida, T. Sakai, A. Matsuzaka, T. Wagner, S. Kanoh, T. Yoshinobu, and M. J. Schoning: Jpn. J. Appl. Phys. 50 (2011).

73 K. Miyamoto, H. Ichimura, T. Wagner, M. J. Schöning, and T. Yoshinobu: Sens. Actuators, B 189 (2013) 240.

74 B. H. Weigl and P. Yager: Sens. Actuators, B 39 (1997) 452.

75 J. P. Brody and P. Yager: Sens. Actuators, A 58 (1997) 13.

76 K. Miyamoto, Y. Hirayama, T. Wagner, M. J. Schöning, and T. Yoshinobu: Electrochim. Acta 113 (2013) 768.

77 C. F. Werner, S. Takenaga, H. Taki, K. Sawada, and M. J. Schoning: Sens. Actuators, B 177 (2013) 745.

78 T. Yoshinobu, H. Ecken, A. B. Md. Ismail, H. Iwasaki, H. Lüth, and M. J. Schöning: Electrochim. Acta 47 (2001) 259.

79 A. Poghossian and M. J. Schöning: Electroanal. 26 (2014) 1197.

80 K. Giebel, C. Bechinger, S. Herminghaus, M. Riedel, P. Leiderer, U. Weiland, and M. Bastmeyer: Biophys. J. 76 (1999) 509. 
81 X. L. Zong, C. S. Wu, X. L. Wu, Y. F. Lu, and P. Wang: Journal of Zhejiang University-Science, B 10 (2009) 860.

82 C. Wu, T. Bronder, A. Poghossian, C. F. Werner, M. Bäcker, and M. J. Schöning: Phys. Status Solidi A 211 (2014) 1423.

83 T. S. Bronder, A. Poghossian, S. Scheja, C. Wu, M. Keusgen, D. Mewes, and M. J. Schöning: ACS Appl. Mater. Interfaces 7 (2015) 20068. 